Homology, Homotopy and Applications, vol.17(2), 2015, pp.235-260

\title{
MODEL STRUCTURES ON IND-CATEGORIES AND THE ACCESSIBILITY RANK OF WEAK EQUIVALENCES
}

\author{
ILAN BARNEA AND TOMER M. SCHLANK
}

(communicated by J. Daniel Christensen)

\begin{abstract}
In a recent paper, we introduced a much weaker and easy to verify structure than a model category, which we called a "weak fibration category." We further showed that an essentially small weak fibration category can be "completed" into a full model category structure on its pro-category, provided the pro-category satisfies a certain two-out-of-three property. In the present paper, we give sufficient intrinsic conditions on a weak fibration category for this two-outof-three property to hold. We apply these results to prove theorems giving sufficient conditions for the finite accessibility of the category of weak equivalences in combinatorial model categories. We apply these theorems to the standard model structure on the category of simplicial sets and deduce that its class of weak equivalences is finitely accessible. The same result on simplicial sets was recently proved also by Raptis and Rosický [RaRo], using different methods.
\end{abstract}

\section{Introduction}

In certain respects, in the algebraic approach to homotopy theory, the basic object of study is a category $\mathcal{C}$ endowed with a class of morphisms $\mathcal{W}$, called weak-equivalences, that should be considered as "isomorphisms honoris causa." If the class of weak-equivalences is well behaved, we say that $(\mathcal{C}, \mathcal{W})$ is a relative category:

Definition 1.1. A relative category is a pair $(\mathcal{C}, \mathcal{W})$, consisting of a category $\mathcal{C}$ and a subcategory $\mathcal{W} \subseteq \mathcal{C}$ that contains all the isomorphisms and satisfies the two-out-ofthree property; $\mathcal{W}$ is called the subcategory of weak-equivalences.

The data of a relative category is enough to define most of the constructions needed in homotopy theory such as mapping spaces, homotopy limits, derived functors, etc. In fact, a relative category is one of the models for the abstract notion of an $(\infty, 1)$-category, which enables one to define these concepts via universal properties (see [BaKa, Lur]).

The first author is supported by the Alexander von Humboldt Professorship of Michael Weiss of the University of Muenster. The second author is supported by the Simons Fellowship in the Department of Mathematics of the Massachusetts Institute of Technology.

Received July 24, 2014, revised February 15, 2015; published on November 18, 2015.

2010 Mathematics Subject Classification: 55U35, 18C35, 55U10.

Key words and phrases: combinatorial model categories, ind-categories, accessibility rank, accessible categories, simplicial sets.

Article available at http://dx.doi.org/10.4310/HHA.2015.v17.n2.a12

Copyright (c) 2015, Ilan Barnea and Tomer M. Schlank. Permission to copy for private use granted. 
Alas, in a relative category, it is in practice very hard to ensure the existence of wanted objects or to carry out any computations. Thus, working effectively in a relative category $(\mathcal{C}, \mathcal{W})$ is usually achieved by adding some extra structure. The most prevalent example is the structure of a model category defined by Quillen in [Qui]. Model categories, albeit very useful, admit quite a "heavy" axiomatization. A model category consists of relative category $(\mathcal{C}, \mathcal{W})$ together with two subcategories $\mathcal{F}, \mathcal{C}$ of of $\mathcal{C}$ called fibrations and cofibrations. The quadruple $(\mathcal{C}, \mathcal{W}, \mathcal{F}, \mathcal{C}$ of $)$ should satisfy many axioms. (We refer the reader to [Hov] for the modern definition of a model category.) The axioms for a model category are often very hard to verify, and furthermore, there are situations in which there is a natural definition of weak equivalences and fibrations; however, the resulting structure is not a model category. (Note that the structure of a model category is determined by the classes of weak equivalences and fibrations, since the class of cofibrations is then determined by a left lifting property.)

In [BaSc2], we introduced a structure that is easier to verify and much weaker than a model category; we called it a "weak fibration category." A weak fibration category consists of a relative category $(\mathcal{C}, \mathcal{W})$ together with one subcategory $\mathcal{F}$ of $\mathcal{C}$ called fibrations, satisfying certain axioms (see Definition 2.12). In [BaSc2], we show that a weak fibration category can be "completed" into a full model category structure on its pro-category, provided it satisfies conditions that we call "pro-admissible" and "homotopically small." The property of being homotopically small is a bit technical and we will not need it here. What is important for us here is that any essentially small weak fibration category is homotopically small.

The property of pro-admissibility is easier to define, and we now bring a definition. Let $(\mathcal{C}, \mathcal{W}, \mathcal{F})$ be a weak fibration category. We say that a morphism in $\operatorname{Pro}(\mathcal{C})$ is in $L w^{\cong}(\mathcal{W})$ if it is isomorphic, as a morphism in $\operatorname{Pro}(\mathcal{C})$, to a natural transformation that is level-wise in $\mathcal{W}$.

Remark 1.2. It is not hard to see that $L w \cong(\mathcal{W})$ is the essential image of $\operatorname{Pro}(\mathcal{W})$ under the natural equivalence $\operatorname{Pro}\left(\mathcal{C}^{\rightarrow}\right) \rightarrow \operatorname{Pro}(\mathcal{C}) \rightarrow$ (where $\mathcal{W}$ is considered as a full subcategory of $\mathcal{C}^{\rightarrow}$ ).

We say that the weak fibration category $(\mathcal{C}, \mathcal{F}, \mathcal{W})$ is pro-admissible if

$$
\left(\operatorname{Pro}(\mathcal{C}), L w^{\cong}(\mathcal{W})\right)
$$

is a relative category (or, in other words, if $L w \cong(\mathcal{W})$ satisfies the two-out-of-three property). From [BaSc2, Theorem 4.18] it easily follows (for more details see Theorem 2.18):

Theorem 1.3. Let $(\mathcal{C}, \mathcal{W}, \mathcal{F})$ be an essentially small pro-admissible weak fibration category. Then there exists a model category structure on $\operatorname{Pro}(\mathcal{C})$ such that:

1. The weak equivalences are $\mathbf{W}:=L w \cong(\mathcal{W})$.

2. The cofibrations are $\mathbf{C}:={ }^{\perp}(\mathcal{F} \cap \mathcal{W})$.

3. The fibrations are maps satisfying the right lifting property with respect to all acyclic fibrations.

Moreover, this model category is $\omega$-cocombinatorial, with $\mathcal{F}$ as the set of generating fibrations and $\mathcal{F} \cap \mathcal{W}$ as the set of generating acyclic fibrations. 
Remark 1.4. 1. Note that by abuse of notation we consider morphisms of $\mathcal{C}$ as morphisms of $\operatorname{Pro}(\mathcal{C})$ indexed by the trivial diagram.

2. A more explicit description of the fibrations in this model structure can be given, but this requires some more definitions. We give the more detailed theorem in the appendix (see Theorem A.7).

3. A model category is said to be cocombinatorial if its opposite category is combinatorial. Combinatorial model categories were introduced by J. H. Smith as model categories that are locally presentable and cofibrantly generated (see, for instance, the appendix of [Lur]). If $\gamma$ is a regular cardinal, we also follow J. H. Smith and call a model category $\gamma$-combinatorial if it is combinatorial and both cofibrations and trivial cofibrations are generated by sets of morphisms having $\gamma$-presentable domains and codomains.

The pro-admissibility condition on a weak cofibration category $\mathcal{C}$, appearing in Theorem 1.3, is not intrinsic to $\mathcal{C}$. It is useful to be able to deduce the pro-admissibility of $\mathcal{C}$ only from conditions on $\mathcal{C}$ itself. One purpose of this paper is to give one possible solution to this problem. This is done in Section 3.

Everything we have discussed so far is completely dualizable. Thus we can define the notion of an ind-admissible weak cofibration category, and show the following:

Theorem 1.5. Let $(\mathcal{M}, \mathcal{W}, \mathcal{C})$ be an essentially small ind-admissible weak cofibration category. Then there exists a model category structure on $\operatorname{Ind}(\mathcal{M})$ such that:

1. The weak equivalences are $\mathbf{W}:=L w^{\simeq}(\mathcal{W})$.

2. The fibrations are $\mathbf{F}=(\mathcal{C} \cap \mathcal{W})^{\perp}$.

3. The cofibrations are maps satisfying the left lifting property with respect to all acyclic fibrations.

Moreover, this model category is $\omega$-combinatorial, with $\mathcal{C}$ as the set of generating cofibrations and $\mathcal{C} \cap \mathcal{W}$ as the set of generating acyclic cofibrations.

Model categories constructed using Theorem 1.5 have some further convenient property, namely, their class of weak equivalences is finitely accessible, when viewed as a full subcategory of the morphism category (we follow the terminology of [AdRo] throughout this paper). This means that it is of the form $\operatorname{Ind}(\mathcal{D})$ for some essentially small category $\mathcal{D}$. This assertion follows from the observation that $L w^{\simeq}(\mathcal{W})$ is the essential image of $\operatorname{Ind}(\mathcal{W})$ under the natural equivalence $\operatorname{Ind}\left(\mathcal{C}^{\rightarrow}\right) \rightarrow \operatorname{Ind}(\mathcal{C}) \rightarrow$, where $\mathcal{W}$ is considered as a full subcategory of $\mathcal{C} \rightarrow$. It then follows that $\operatorname{Ind}(\mathcal{W})$ is a full subcategory of $\operatorname{Ind}\left(\mathcal{C}^{\rightarrow}\right)$, and thus $\operatorname{Ind}(\mathcal{W}) \simeq L w \cong(\mathcal{W})$.

In $[\mathbf{B a S c 2}]$ we have applied (a generalization of) Theorem 1.3 to a specific weak fibration category (namely, the category of simplicial sheaves over a Grothendieck site, where the weak equivalences and the fibrations are local in the sense of Jardine) to obtain a novel model structure in its pro-category. In this paper we also consider an application of Theorem 1.3 (or rather of its dual version, Theorem 1.5), but in a reverse direction. Namely, we begin with an $\omega$-combinatorial model category $\mathcal{M}$ and ask whether the model structure on $\mathcal{M}$ is induced, via Theorem 1.5, from a weak cofibration structure on its full subcategory of finitely presentable objects. The main conclusion we wish to deduce from this is the finite accessibility of the class of weak equivalences in $\underline{\mathcal{M}}$, as explained above. 
While we were writing the first draft of this paper, Raptis and Rosický published a paper with some related results $[\mathbf{R a R o}]$. In their paper, Raptis and Rosický mention that while the class of weak equivalences in any combinatorial model category is known to be accessible, the known estimates for the accessibility rank are generally not the best possible. In their paper, they prove theorems giving estimates for the accessibility rank of weak equivalences in various cases. Their main application is to the standard model structure on simplicial sets. They show that the class of weak equivalences in this model structure is finitely accessible.

The purpose of this paper is the same, as well as the main example. Namely, we prove theorems giving estimates for the accessibility rank of weak equivalences in various cases, and our main example is the category of simplicial sets on which we achieve a similar estimate as [RaRo]. However, our theorems, as well as the methods of proof, are completely different. Since our basic tool is based on applying Theorem 1.5 as explained above, our estimates only concern finite accessibility. We do believe, however, that Theorem 1.5, and thus also our results here, can be generalized to an arbitrary cardinal instead of $\omega$. On the other hand, our theorems apply also in cases where the theorems in $[\mathbf{R a R o}]$ do not.

We will now state our main results. For this, we first need a definition:

Definition 1.6. Let $(\mathcal{C}, \mathcal{W})$ be a relative category. A map $f: A \rightarrow B$ in $\mathcal{C}$ will be called right proper, if for every pull-back square of the form

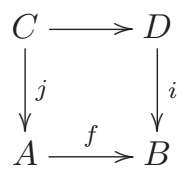

such that $i$ is a weak equivalence, the map $j$ is also a weak equivalence.

We can now state our first criterion for the finite accessibility of the category of weak equivalences (see Theorem 4.4):

Theorem 1.7. Let $(\underline{\mathcal{M}}, \underline{\mathcal{W}}, \underline{\mathcal{F}}, \underline{\mathcal{C}})$ be an $\omega$-combinatorial left proper model category. Let $\mathcal{M}$ denote the full subcategory of $\underline{\mathcal{M}}$ spanned by the finitely presentable objects.

Suppose we are given a cylinder object in $\mathcal{M}$, that is, for every object $B$ of $\mathcal{M}$ we are given a factorization in $\mathcal{M}$ of the fold map $B \sqcup B \rightarrow B$ into a cofibration followed by a weak equivalence:

$$
B \sqcup B \stackrel{\left(i_{0}, i_{1}\right)}{\longrightarrow} I \otimes B \stackrel{p}{\rightarrow} B .
$$

(Note that we are not assuming any simplicial structure; $I \otimes B$ is just a suggestive notation.)

We make the following further assumptions:

1. The category $\mathcal{M}$ has finite limits.

2. Every object in $\mathcal{M}$ is cofibrant. 
3. For every morphism $f: A \rightarrow B$ in $\mathcal{M}$ the map $B \coprod_{A}(I \otimes A) \rightarrow B$, induced by the commutative square

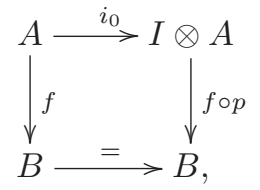

is a right proper map in $(\mathcal{M}, \mathcal{W})$.

Then the full subcategory of the morphism category of $\underline{\mathcal{M}}$, spanned by the class of weak equivalences, is finitely accessible.

Our second criterion can be shown using the first one (see Theorem 4.5):

Theorem 1.8. Let $(\underline{\mathcal{M}}, \underline{\mathcal{W}}, \underline{\mathcal{F}}, \underline{\mathcal{C}})$ be an $\omega$-combinatorial left proper model category. Let $\mathcal{M}$ denote the full subcategory of $\mathcal{M}$ spanned by the finitely presentable objects. Assume that the category $\mathcal{M}$ has finite limits, and let $*$ denote the terminal object in $\mathcal{M}$.

Suppose we are given a factorization in $\mathcal{M}$ of the fold $\operatorname{map} * \sqcup * \rightarrow *$ into a cofibration followed by a weak equivalence:

$$
* \sqcup * \rightarrow I \rightarrow * .
$$

We make the following further assumptions:

1. For every morphism $Y \rightarrow B$ in $\mathcal{M}$, the functor

$$
Y \times_{B}(-): \mathcal{M} / B \rightarrow \mathcal{M}
$$

commutes with finite colimits.

2. Every object in $\mathcal{M}$ is cofibrant.

3. For every object $B$ in $\mathcal{M}$, the functor

$$
B \times(-): \mathcal{M} \rightarrow \mathcal{M}
$$

preserves cofibrations and weak equivalences.

Then the full subcategory of the morphism category of $\underline{\mathcal{M}}$, spanned by the class of weak equivalences, is finitely accessible.

It is not hard to verify that the standard model structure on the category of simplicial sets satisfies the hypothesis of the previous theorem (see Theorem 4.6). Thus, we obtain the following:

Theorem 1.9. The full subcategory of the morphism category of $\mathcal{S}$, spanned by the class of weak equivalences, is finitely accessible.

As mentioned above, Theorem 1.9 was also proved in [RaRo], using different methods. In the appendix, we prove some results that might shed some light as to possible connections between the approach taken in this paper, and that of Raptis and Rosický. To prove these results, we will need to present the more detailed version of Theorem 1.5 (see Theorem A.7). 


\subsection{Organization of the paper}

In Section 2, we give a short review of the necessary background on pro-categories and model structures on them. Everything in this section dualizes easily to indcategories. In Section 3, we prove a theorem giving sufficient intrinsic conditions for the pro-admissibility of a weak fibration category. We also define an auxiliary notion that generalizes the notion of a model category. The results and definitions of Section 3 will be used in Section 4, where we prove the main results of this paper, namely, a series of criteria for the finite accessibility of the category of weak equivalences.

\section{Acknowledgments}

We would like to thank Yonatan Harpaz for useful conversations. We also thank Dmitri Pavlov for pointing out the relation of our work to the work of Raptis and Rosický [RaRo], and Geoffroy Horel for Remark 3.2. Finally, we would like to thank the referee for his useful comments.

\section{Preliminaries: model structures on pro-categories}

In this section, we review the necessary background of model structures on procategories. We state the results without proof, for later reference. For proofs and more information, the reader is referred to [ArMa, Isa1, BaSc1, BaSc2]. All these results are easily dualized to the case of ind-categories.

\subsection{Pro-categories}

Definition 2.1. A category $I$ is called cofiltered if the following conditions are satisfied:

1. The category $I$ is non-empty.

2. For every pair of objects $s, t \in I$, there exists an object $u \in I$, together with morphisms $u \rightarrow s$ and $u \rightarrow t$.

3. For every pair of morphisms $f, g: s \rightarrow t$ in $I$, there exists a morphism $h: u \rightarrow s$ in $I$ such that $f \circ h=g \circ h$.

A category is called small if it has only a set of objects and a set of morphisms.

Definition 2.2. Let $\mathcal{C}$ be a category. The category $\operatorname{Pro}(\mathcal{C})$ has as objects all diagrams in $\mathcal{C}$ of the form $I \rightarrow \mathcal{C}$ such that $I$ is small and cofiltered (see Definition 2.1). The morphisms are defined by the formula

$$
\operatorname{Hom}_{\operatorname{Pro}(\mathcal{C})}(X, Y):=\lim _{s} \operatorname{colim}_{t} \operatorname{Hom}_{\mathcal{C}}\left(X_{t}, Y_{s}\right)
$$

Composition of morphisms is defined in the obvious way.

Thus, if $X: I \rightarrow \mathcal{C}$ and $Y: J \rightarrow \mathcal{C}$ are objects in Pro $(\mathcal{C})$, providing a morphism $X \rightarrow Y$ means specifying for every $s$ in $J$ an object $t$ in $I$ and a morphism $X_{t} \rightarrow Y_{s}$ in $\mathcal{C}$. These morphisms should of course satisfy some compatibility condition. In particular, if the indexing categories are equal, $I=J$, any natural transformation $X \rightarrow Y$ gives rise to a morphism $X \rightarrow Y$ in $\operatorname{Pro}(C)$. More generally, if $p: J \rightarrow I$ is a functor, and $\phi: p^{*} X:=X \circ p \rightarrow Y$ is a natural transformation, then the pair 
$(p, \phi)$ determines a morphism $\nu_{p, \phi}: X \rightarrow Y$ in $\operatorname{Pro}(C)$ (for every $s$ in $J$, we take the morphism $\left.\phi_{s}: X_{p(s)} \rightarrow Y_{s}\right)$. In particular, taking $Y=p^{*} X$ and $\phi$ to be the identity natural transformation, we see that $p$ determines a morphism $\nu_{p, X}: X \rightarrow p^{*} X$ in $\operatorname{Pro}(C)$.

The word pro-object refers to objects of pro-categories. A simple pro-object is one indexed by the category with one object and one (identity) map. Note that for any category $\mathcal{C}, \operatorname{Pro}(\mathcal{C})$ contains $\mathcal{C}$ as the full subcategory spanned by the simple objects.

Definition 2.3. Let $p: J \rightarrow I$ be a functor between small categories. The functor $p$ is said to be (left) cofinal if for every $i$ in $I$ the over category $p_{/ i}$ is non-empty and connected.

Cofinal functors play an important role in the theory of pro-categories mainly because of the following well-known lemma (see, for example, $[\mathbf{A r M a}]$ ):

Lemma 2.4. Let $p: J \rightarrow I$ be a cofinal functor between small cofiltered categories, and let $X: I \rightarrow \mathcal{C}$ be an object in $\operatorname{Pro}(\mathcal{C})$. Then the morphism in $\operatorname{Pro}(\mathcal{C})$ that $p$ induces, $\nu_{p, X}: X \rightarrow p^{*} X$, is an isomorphism.

The following lemma can be found in [ArMa, Appendix 3.2]. See also [BaSc1, Corollary 3.26] for a stronger result.

Lemma 2.5. Every morphism in $\operatorname{Pro}(\mathcal{C})$ is isomorphic, in the category of morphisms in $\operatorname{Pro}(\mathcal{C})$, to a morphism that comes from a natural transformation (that is, to a morphism of the form $\nu_{i d, \phi}$, where $\phi$ is a natural transformation).

Definition 2.6. Let $\mathcal{C}$ be a category, $M \subseteq \operatorname{Mor}(\mathcal{C})$ a class of morphisms in $\mathcal{C}, I$ a small category, and $F: X \rightarrow Y$ a morphism in $\mathcal{C}^{I}$. Then $F$ will be called a level-wise $M$-map if for every $i \in I$ the morphism $X_{i} \rightarrow Y_{i}$ is in $M$. We will denote this by $F \in L w(M)$.

Definition 2.7. Let $\mathcal{C}$ be a category, and let $M \subseteq \operatorname{Mor}(\mathcal{C})$ be a class of morphisms in $\mathcal{C}$. Denote by

1. ${ }^{\perp} M$ the class of morphisms in $\mathcal{C}$ having the left lifting property w.r.t. any morphism in $M$;

2. $M^{\perp}$ the class of morphisms in $\mathcal{C}$ having the right lifting property w.r.t. any morphism in $M$;

3. $L w^{\simeq}(M)$ the class of morphisms in $\operatorname{Pro}(\mathcal{C})$ that are isomorphic to a morphism that comes from a natural transformation which is a level-wise $M$-map.

Everything we have done so far (and throughout this paper) is completely dualizable. Thus, we can define the following:

Definition 2.8. A category $I$ is called filtered if the following conditions are satisfied:

1. The category $I$ is non-empty.

2. For every pair of objects $s, t \in I$, there exists an object $u \in I$, together with morphisms $s \rightarrow u$ and $t \rightarrow u$.

3. For every pair of morphisms $f, g: s \rightarrow t$ in $I$, there exists a morphism $h: t \rightarrow u$ in $I$ such that $h \circ f=h \circ g$. 
The dual to the notion of a pro-category is the notion of an ind-category:

Definition 2.9. Let $\mathcal{C}$ be a category. The category $\operatorname{Ind}(\mathcal{C})$ has as objects all diagrams in $\mathcal{C}$ of the form $I \rightarrow \mathcal{C}$ such that $I$ is small and filtered (see Definition 2.8). The morphisms are defined by the formula

$$
\operatorname{Hom}_{\operatorname{Pro}(\mathcal{C})}(X, Y):=\lim _{s} \operatorname{colim}_{t} \operatorname{Hom}_{\mathcal{C}}\left(X_{s}, Y_{t}\right)
$$

Composition of morphisms is defined in the obvious way.

Clearly for every category $\mathcal{C}$ we have a natural isomorphism of categories: $\operatorname{Ind}(\mathcal{C})^{o p} \cong$ $\operatorname{Pro}\left(\mathcal{C}^{o p}\right)$.

We are not going to write the dual to every definition or theorem explicitly, only in certain cases.

\subsection{From a weak fibration category to a model category}

We now present the definition of a weak fibration category, after two preliminary definitions:

Definition 2.10. Let $\mathcal{C}$ be a category, and let $M, N$ be classes of morphisms in $\mathcal{C}$. We will denote by $\operatorname{Mor}(\mathcal{C})=M \circ N$ the assertion that every map $A \rightarrow B$ in $\mathcal{C}$ can be factored as $A \stackrel{f}{\rightarrow} C \stackrel{g}{\rightarrow} B$, where $f$ is in $N$ and $g$ is in $M$.

Definition 2.11. Let $\mathcal{C}$ be a category with finite limits, and let $\mathcal{M} \subseteq \mathcal{C}$ be a subcategory. We say that $\mathcal{M}$ is closed under base change if whenever we have a pull-back square

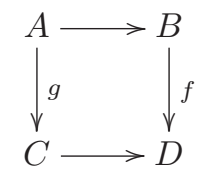

such that $f$ is in $\mathcal{M}$, then $g$ is in $\mathcal{M}$.

Definition 2.12. A weak fibration category is a category $\mathcal{C}$ with an additional structure of two subcategories

$$
\mathcal{F}, \mathcal{W} \subseteq \mathcal{C}
$$

that contain all the isomorphisms, such that the following conditions are satisfied:

1. $\mathcal{C}$ has all finite limits.

2. $\mathcal{W}$ has the two-out-of-three property.

3. The subcategories $\mathcal{F}$ and $\mathcal{F} \cap \mathcal{W}$ are closed under base change.

4. $\operatorname{Mor}(\mathcal{C})=\mathcal{F} \circ \mathcal{W}$.

The maps in $\mathcal{F}$ are called fibrations, and the maps in $\mathcal{W}$ are called weak equivalences.

Remark 2.13. The notion of a weak fibration category is closely related other notions considered previously in the literature such as a "category of fibrant objects" [Bro], a "fibration category" [Bau], an "Anderson-Brown-Cisinski fibration category" [Rad], and more. These notions were introduced as a more flexible structure than a model category in which to do abstract homotopy theory. 
Definition 2.14. A relative category is a pair $(\mathcal{C}, \mathcal{W})$, consisting of a category $\mathcal{C}$ and a subcategory $\mathcal{W} \subseteq \mathcal{C}$ that contains all the isomorphisms and satisfies the two-out-ofthree property; $\mathcal{W}$ is called the subcategory of weak equivalences.

Remark 2.15. Any weak fibration category is naturally a relative category when ignoring the fibrations.

Definition 2.16. We will denote by $\rightarrow$ the category consisting of two objects and one non-identity morphism between them. Thus, if $\mathcal{C}$ is any category, the functor category $\mathcal{C} \rightarrow$ is just the category of morphisms in $\mathcal{C}$.

Definition 2.17. A relative category $(\mathcal{C}, \mathcal{W})$ will be called

1. pro-admissible, if $L w^{\cong}(\mathcal{W}) \subseteq \operatorname{Pro}(\mathcal{C}) \rightarrow$ satisfies the two-out-of-three property;

2. ind-admissible, if $L w \cong(\mathcal{W}) \subseteq \operatorname{Ind}(\mathcal{C}) \rightarrow$ satisfies the two-out-of-three property;

3. admissible, if it both pro- and ind-admissible.

The following theorem is almost a special case of [BaSc2, Theorem 4.18]:

Theorem 2.18. Let $(\mathcal{C}, \mathcal{W}, \mathcal{F})$ be an essentially small pro-admissible weak fibration category. Then there exists a model category structure on $\operatorname{Pro}(\mathcal{C})$ such that:

1. The weak equivalences are $\mathbf{W}:=L w \cong(\mathcal{W})$.

2. The cofibrations are $\mathbf{C}:={ }^{\perp}(\mathcal{F} \cap \mathcal{W})$.

3. The fibrations are maps satisfying the right lifting property with respect to all acyclic cofibrations.

Moreover, this model category is $\omega$-cocombinatorial, with $\mathcal{F}$ as the set of generating fibrations and $\mathcal{F} \cap \mathcal{W}$ as the set of generating acyclic fibrations.

Remark 2.19. The definition of a model category that we refer to in Theorem 2.18 is the one used in $[\mathbf{H o v}]$. In particular, we require functorial factorizations. This is a stronger conclusion then that of [BaSc2, Theorem 4.18] and is achieved because we assume that $\mathcal{C}$ is essentially small. Notice that we did not require functorial factorizations in the definition of a weak fibration category. Note also that we only required the existence of finite limits in the definition of a weak fibration category, while in $\operatorname{Pro}(\mathcal{C})$ we do get the existence of arbitrary limits and colimits.

Proof. Most of the proof goes exactly like the proof of [BaSc2, Theorem 4.18] the only difference is that we can use [BaSc2, Proposition 3.18] instead of [BaSc2, Proposition 3.17], and thus obtain functorial factorizations.

It only remains to show that $\operatorname{Pro}(\mathcal{C})$ is $\omega$-cocombinatorial, with set of generating fibrations $\mathcal{F}$ and set of generating acyclic fibrations $\mathcal{F} \cap \mathcal{W}$. The category $\mathcal{C}$ has finite limits, so $\mathcal{C}^{o p}$ has finite colimits. By the results of $[\mathbf{A d R o}]$, the category $\operatorname{Ind}\left(\mathcal{C}^{o p}\right) \cong$ $\operatorname{Pro}(\mathcal{C})^{o p}$ is locally presentable and every object of $\mathcal{C}^{o p}$ is $\omega$-presentable in $\operatorname{Ind}\left(\mathcal{C}^{o p}\right)$. It thus remains to show that

$$
\mathbf{C}={ }^{\perp}(\mathcal{F} \cap \mathcal{W}),(\mathbf{C} \cap \mathbf{W})={ }^{\perp} \mathcal{F},
$$

but this was shown in [BaSc2, Theorem 4.18]. 
The dual to the notion of a weak fibration category is a weak cofibration category. Namely, a weak cofibration category is a category $\mathcal{M}$ together with two subcategories, $\mathcal{C}$ and $\mathcal{W}$, such that $\left(\mathcal{M}^{o p}, \mathcal{C}^{o p}, \mathcal{W}^{o p}\right)$ is a weak fibration category. The following is a dual formulation of Theorem 2.18:

Theorem 2.20. Let $(\mathcal{M}, \mathcal{W}, \mathcal{C})$ be an essentially small ind-admissible weak cofibration category. Then there exists a model category structure on $\operatorname{Ind}(\mathcal{M})$ such that:

1. The weak equivalences are $\mathbf{W}:=L w \cong(\mathcal{W})$.

2. The fibrations are $\mathbf{F}=(\mathcal{C} \cap \mathcal{W})^{\perp}$.

3. The cofibrations are maps satisfying the left lifting property with respect to all acyclic fibrations.

Moreover, this model category is $\omega$-combinatorial, with $\mathcal{C}$ as the set of generating cofibrations and $\mathcal{C} \cap \mathcal{W}$ as the set of generating acyclic cofibrations.

\section{Proper morphisms}

\subsection{A criterion for the two-out-of-three property}

The pro-admissibility condition on a relative category $\mathcal{C}$, appearing in Theorem 2.18, is not intrinsic to $\mathcal{C}$ (see Definition 2.17). It is useful to be able to deduce the proadmissibility of $\mathcal{C}$ only from conditions on $\mathcal{C}$ itself. In this subsection, we give one possible solution to this problem. The idea is a very straightforward generalization of an idea of Isaksen [Isa1, Section 3].

Definition 3.1. Let $(\mathcal{C}, \mathcal{W})$ be a relative category. A map $f: A \rightarrow B$ in $\mathcal{C}$ will be called

1. left proper, if for every push-out square of the form

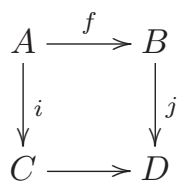

such that $i$ is a weak equivalence, the map $j$ is also a weak equivalence; and

2. right proper, if for every pull back square of the form

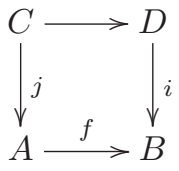

such that $i$ is a weak equivalence, the map $j$ is also a weak equivalence.

We denote by $L P$ the class of left proper maps in $\mathcal{C}$ and by $R P$ the class of right proper maps in $\mathcal{C}$.

Remark 3.2. The notion of a right proper map is related to the notion of a sharp map defined by Rezk in [Rez]. A sharp map is a map such that all its base changes are right proper. In other words, the class of sharp maps is the largest class of maps that is contained in the right proper maps and is closed under base change (see 
Definition 2.11). A sharp map is called a weak fibration by Cisinski and a fibrillation by Barwick and Kan.

Example 3.3. Let $\mathcal{M}$ be a model category. Then:

1. Every acyclic cofibration in $\mathcal{M}$ is a left proper map in $(\mathcal{M}, \mathcal{W})$.

2. Every acyclic fibration in $\mathcal{M}$ is a right proper map in $(\mathcal{M}, \mathcal{W})$.

3. The model category $\mathcal{M}$ is left proper iff every cofibration in $\mathcal{M}$ is a left proper map in $(\mathcal{M}, \mathcal{W})$.

4. The model category $\mathcal{M}$ is right proper iff every fibration in $\mathcal{M}$ is a right proper map in $(\mathcal{M}, \mathcal{W})$.

Definition 3.4. Let $(\mathcal{C}, \mathcal{W})$ be a relative category. Then $(\mathcal{C}, \mathcal{W})$ will be said to have proper factorizations, if the following hold:

1. $\operatorname{Mor}(\mathcal{C})=R P \circ L P$.

2. $\operatorname{Mor}(\mathcal{C})=R P \circ \mathcal{W}$.

3. $\operatorname{Mor}(\mathcal{C})=\mathcal{W} \circ L P$.

Lemma 3.5. Let $\mathcal{M}$ be a proper model category. Then the relative category $(\mathcal{M}, \mathcal{W})$ has proper factorizations.

Proof. 1. $\operatorname{Mor}(\mathcal{M})=R P \circ L P$ is shown by factoring every map into a cofibration followed by an acyclic fibration (see Example 3.3).

2. $\operatorname{Mor}(\mathcal{C})=R P \circ \mathcal{W}$ is shown by factoring every map into an acyclic cofibration followed by a fibration (see Example 3.3).

3. $\operatorname{Mor}(\mathcal{C})=\mathcal{W} \circ L P$ is shown by factoring every map into a cofibration followed by an acyclic fibration (see Example 3.3).

The following is shown in [Isa1, Lemma 3.2] (see also [Isa1, Remark 3.3]):

Lemma 3.6. Let $\mathcal{C}$ be a category, and let $N$ and $M$ be classes of morphisms in $\mathcal{C}$, such that $\operatorname{Mor}(\mathcal{C})=M \circ N$. Let $T$ be a cofiltered category, and let $f:\left\{X_{t}\right\}_{t \in T} \rightarrow\left\{Y_{t}\right\}_{t \in T}$ be a natural transformation, that is, a map in the functor category $\mathcal{C}^{T}$. Suppose that $f$ is an isomorphism as a map in $\operatorname{Pro}(\mathcal{C})$ (or $\operatorname{Ind}(\mathcal{C})$ ).

Then there exist a cofiltered category $J$, a cofinal functor $p: J \rightarrow T$, and a factorization $p^{*} X \stackrel{g}{\rightarrow} H_{f} \stackrel{h}{\rightarrow} p^{*} Y$ of $p^{*} f: p^{*} X \rightarrow p^{*} Y$ in the category $C^{J}$ such that $h$ is a level-wise $\mathcal{M}$ map, $g$ is a level-wise $N$ map, and $g$, $h$ are isomorphisms as maps in $\operatorname{Pro}(\mathcal{C})$ (or $\operatorname{Ind}(\mathcal{C}))$.

The following proposition is our main motivation for introducing the concepts of left and right proper morphisms:

Proposition 3.7. Let $(\mathcal{C}, \mathcal{W})$ be a relative category, and let $X \stackrel{f}{\rightarrow} Y \stackrel{g}{\rightarrow} Z$ be a pair of composable morphisms in $\operatorname{Pro}(\mathcal{C})$ (or $\operatorname{Ind}(\mathcal{C})$ ). Then:

1. If $\mathcal{C}$ has finite limits and colimits, and $\operatorname{Mor}(\mathcal{C})=R P \circ L P$, then $f, g \in L w \cong(\mathcal{W})$ implies that $g \circ f \in L w \cong(\mathcal{W})$. 
2. If $\mathcal{C}$ has finite limits, and $\operatorname{Mor}(\mathcal{C})=R P \circ \mathcal{W}$, then $g, g \circ f \in L w \cong(\mathcal{W})$ implies that $f \in L w^{\cong}(\mathcal{W})$.

3. If $\mathcal{C}$ has finite colimits, and $\operatorname{Mor}(\mathcal{C})=\mathcal{W} \circ L P$, then $f, g \circ f \in L w \cong(\mathcal{W})$ implies that $g \in L w \cong(\mathcal{W})$.

Proof. For simplicity of writing, we only examine the $\operatorname{Pro}(\mathcal{C})$ case.

We show 1. The proof is a straightforward generalization of the proof of [Isa1, Lemma 3.5].

Since $f, g \in L w^{\simeq}(\mathcal{W})$, there exists a diagram in $\operatorname{Pro}(\mathcal{C})$,

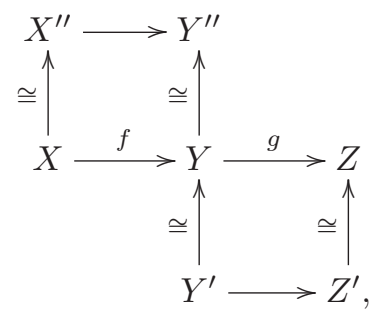

such that the vertical maps are isomorphisms in $\operatorname{Pro}(\mathcal{C})$ and such that $Y^{\prime} \rightarrow Z^{\prime}$ is a natural transformation indexed by $I$ that is level-wise in $\mathcal{W}$ and $X^{\prime \prime} \rightarrow Y^{\prime \prime}$ is a natural transformation indexed by $J$ that is level-wise in $\mathcal{W}$.

Let $Y^{\prime} \stackrel{\cong}{\rightarrow} Y^{\prime \prime}$ denote the composition $Y^{\prime} \cong$ (but not necessarily a level-wise isomorphism). It follows from [ArMa, Appendix 3.2] that there exists a cofiltered category $K$, cofinal functors $p: K \rightarrow I$ and $q: K \rightarrow J$, and a map in $\mathcal{C}^{K}$,

$$
q^{*} Y^{\prime} \rightarrow p^{*} Y^{\prime \prime}
$$

such that there is a commutative diagram in $\operatorname{Pro}(\mathcal{C})$,

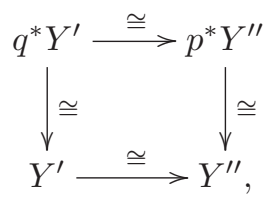

with all maps isomorphisms. Thus, we have a diagram in $\mathcal{C}^{K}$,

$$
p^{*} X^{\prime \prime} \rightarrow p^{*} Y^{\prime \prime} \cong q^{*} Y^{\prime} \rightarrow q^{*} Z^{\prime},
$$

such that the first and last maps are level-wise in $\mathcal{W}$ and the middle map is an isomorphism as a map in $\operatorname{Pro}(\mathcal{C})$ (but not necessarily a level-wise isomorphism).

Since $\operatorname{Mor}(\mathcal{C})=R P \circ L P$, we get by Lemma 3.6, applied for $M=R P$ and $N=$ $L P$, that after pulling back by a cofinal functor $T \rightarrow K$ we obtain a diagram in $\mathcal{C}^{T}$,

$$
A \rightarrow B \stackrel{\cong}{\cong} E C \rightarrow D,
$$

such that the first and last maps are level-wise in $\mathcal{W}$, the second map is level-wise right proper and an isomorphism in $\operatorname{Pro}(\mathcal{C})$, and the third map is level-wise left proper and an isomorphism in $\operatorname{Pro}(\mathcal{C})$. 
By Corollary 3.19 of [BaSc1], since $\mathcal{C}$ has finite limits and colimits, the pull-back and push-out in $\operatorname{Pro}(\mathcal{C})$ of a diagram in $\mathcal{C}^{T}$ can be computed level-wise. We thus get the following diagram in $\mathcal{C}^{T}$ :

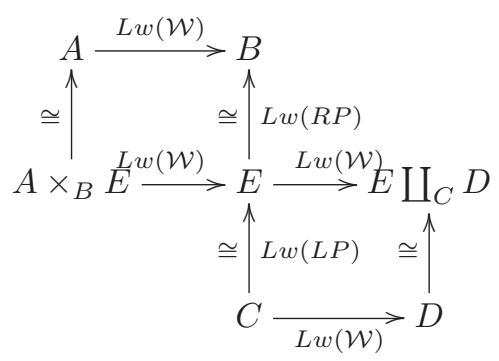

where $\cong$ indicates an isomorphism in $\operatorname{Pro}(\mathcal{C})$.

It follows that the composition

$$
A \times_{B} E \stackrel{L w(\mathcal{W})}{\longrightarrow} E \stackrel{L w(\mathcal{W})}{\longrightarrow} E \coprod_{C} D
$$

is a level-wise $\mathcal{W}$ map that is isomorphic, as a map in $\operatorname{Pro}(\mathcal{C})$, to the composition $g \circ f$. Thus, we obtain that $g \circ f \in L w \cong(\mathcal{W})$.

It is not hard to show 2 and 3 using the same type of generalization to the proof of [Isa1, Lemma 3.6].

Corollary 3.8. Let $(\mathcal{C}, \mathcal{W})$ be a relative category that has finite limits and colimits and proper factorizations. Then $(\mathcal{C}, \mathcal{W})$ is admissible (see Definition 2.17). In particular, if $\mathcal{C}$ is a proper model category, then $(\mathcal{C}, \mathcal{W})$ is admissible.

\subsection{Almost model categories}

Corollary 3.8 gives sufficient conditions for the admissibility of a relative category and, in particular, of a weak (co)fibration category. However, in some interesting examples these conditions are too restrictive. Namely, in some situations there is a natural mapping cylinder factorization (see the proof of Theorem 4.4) that can be shown to give factorizations of the forms $\operatorname{Mor}(\mathcal{M})=R P \circ L P$ and $\operatorname{Mor}(\mathcal{M})=$ $\mathcal{W} \circ L P$ but not $\operatorname{Mor}(\mathcal{M})=R P \circ \mathcal{W}$. We will therefore need to use an auxiliary notion that is more general than a model category, which we call an almost model category.

Definition 3.9. An almost model category is a quadruple $(\mathcal{M}, \mathcal{W}, \mathcal{F}, \mathcal{C})$ satisfying all the axioms of a model category, except (maybe) the two-out-of-three property for $\mathcal{W}$. More precisely, an almost model category satisfies the following:

1. $\mathcal{M}$ is complete and cocomplete.

2. $\mathcal{W}$ is a class of morphisms in $\mathcal{M}$ that is closed under retracts.

3. $\mathcal{F}, \mathcal{C}$ are subcategories of $\mathcal{M}$ that are closed under retracts.

4. $\mathcal{C} \cap \mathcal{W} \subseteq{ }^{\perp} \mathcal{F}$ and $\mathcal{C} \subseteq{ }^{\perp}(\mathcal{F} \cap \mathcal{W})$.

5. There exist functorial factorizations in $\mathcal{M}$ into a map in $\mathcal{C} \cap \mathcal{W}$ followed by a map in $\mathcal{F}$, and into a map in $\mathcal{C}$ followed by a map in $\mathcal{F} \cap \mathcal{W}$. 
The following lemma can be proven just as in the case of model categories (see, for example, [Hov, Lemma 1.1.10]):

Lemma 3.10. In an almost model category $(\mathcal{M}, \mathcal{W}, \mathcal{F}, \mathcal{C})$ we have:

1. $\mathcal{C} \cap \mathcal{W}={ }^{\perp} \mathcal{F}$.

2. $\mathcal{C}={ }^{\perp}(\mathcal{F} \cap \mathcal{W})$.

3. $\mathcal{F} \cap \mathcal{W}=\mathcal{C}^{\perp}$.

4. $\mathcal{F}=(\mathcal{C} \cap \mathcal{W})^{\perp}$.

Definition 3.11. A relative category $(\mathcal{C}, \mathcal{W})$ will be called almost pro-admissible, if $L w^{\simeq}(\mathcal{W}) \subseteq \operatorname{Pro}(\mathcal{C}) \rightarrow$ satisfies the following portion of the two-out-of-three property:

For every pair of composable morphisms in $\operatorname{Pro}(\mathcal{C}): X \stackrel{f}{\rightarrow} Z \stackrel{g}{\rightarrow} Y$ we have:

1. If $f, g$ belong to $L w \cong(\mathcal{W})$, then $g \circ f \in L w^{\cong}(\mathcal{W})$.

2. If $g, g \circ f$ belong to $L w \cong(\mathcal{W})$, then $f \in L w^{\cong}(\mathcal{W})$.

We now prove the following generalization of Theorem 2.18:

Theorem 3.12. Let $(\mathcal{C}, \mathcal{W}, \mathcal{F})$ be an essentially small almost pro-admissible weak fibration category. Then there exists an almost model category structure on $\operatorname{Pro}(\mathcal{C})$ such that:

1. The weak equivalences are $\mathbf{W}:=L w^{\cong}(\mathcal{W})$.

2. The cofibrations are $\mathbf{C}:={ }^{\perp}(\mathcal{F} \cap \mathcal{W})$.

3. The fibrations are maps satisfying the right lifting property with respect to all acyclic cofibrations.

Furthermore, we have $\mathbf{C} \cap \mathbf{W}={ }^{\perp} \mathcal{F}$.

Proof. This is very much like the proof of Theorem 2.18, which is based on [BaSc2, Theorem 4.18]. Going over the proof of [BaSc2, Theorem 4.18] we find that we can show all the axioms of a model category for $\operatorname{Pro}(\mathcal{C})$, except the two-out-of-three property for $L w \cong(\mathcal{W})$, using only the fact that $\mathcal{C}$ is almost pro-admissible. (In fact, the only place where we use the fact that $L w^{\simeq}(\mathcal{W})$ satisfies the two-out-of-three property is in the last part of the proof, where we only use the portion of the two-out-of-three property given in Definition 3.11.)

We can dualize the above:

Definition 3.13. A relative category $(\mathcal{C}, \mathcal{W})$ will be called almost ind-admissible, if $L w \simeq(\mathcal{W}) \subseteq \operatorname{Ind}(\mathcal{C}) \rightarrow$ satisfies the following portion of the two-out-of-three property:

For every pair of composable morphisms in $\operatorname{Ind}(\mathcal{C}): X \stackrel{f}{\rightarrow} Z \stackrel{g}{\rightarrow} Y$, we have:

1. If $f, g$ belong to $L w^{\cong}(\mathcal{W})$, then $g \circ f \in L w^{\cong}(\mathcal{W})$.

2. If $f, g \circ f$ belong to $L w \cong(\mathcal{W})$, then $g \in L w \cong(\mathcal{W})$.

Theorem 3.14. Let $(\mathcal{M}, \mathcal{W}, \mathcal{C})$ be an essentially small almost ind-admissible weak cofibration category. Then there exists an almost model category structure on $\operatorname{Ind}(\mathcal{M})$ such that:

1. The weak equivalences are $\mathbf{W}:=L w \cong(\mathcal{W})$. 
2. The fibrations are $\mathbf{F}:=(\mathcal{C} \cap \mathcal{W})^{\perp}$.

3. The cofibrations are maps satisfying the left lifting property with respect to all acyclic fibrations.

Furthermore, we have $\mathbf{F} \cap \mathbf{W}=\mathcal{C}^{\perp}$.

\section{Criteria for finite accessibility}

In this last section, we will state our main results of this paper-namely, a series of criteria for the finite accessibility of the category of weak equivalences. The criteria are stated in a decreasing level of generality (each criterion being an application or a special case of the previous one) but in an increasing level of convenience of verification and applicability. Our only example in this paper is the category of simplicial sets, which is an example of applying the third and last criterion. However, the authors are aware of an example where the second criterion applies but not the third. This is a non-standard model structure on the category of chain complexes of modules over a ring and will be treated in a future paper.

Definition 4.1. A category is called finitely accessible if it has filtered colimits and there is a small set of finitely presentable objects that generates it under filtered colimits.

The following lemma explains the relevance of Theorem 2.20 to the finite accessibility of the category of weak equivalences:

Lemma 4.2. Let $(\mathcal{M}, \mathcal{W}, \mathcal{C})$ be an essentially small ind-admissible weak cofibration category. Consider the model structure induced on $\operatorname{Ind}(\mathcal{M})$ by Theorem 2.20. Then the full subcategory of $\operatorname{Ind}(\mathcal{M}) \rightarrow$, spanned by the class of weak equivalences, is finitely accessible (see Definition 4.1).

Proof. We need to show that $L w \cong(\mathcal{W})$ is of the form $\operatorname{Ind}(\mathcal{D})$ for some essentially small category $\mathcal{D}$. This follows from the observation that $L w^{\cong}(\mathcal{W})$ is the essential image of $\operatorname{Ind}(\mathcal{W})$ under the natural equivalence $\operatorname{Ind}\left(\mathcal{C}^{\rightarrow}\right) \rightarrow \operatorname{Ind}(\mathcal{C}) \rightarrow$, where $\mathcal{W}$ is considered as a full subcategory of $\mathcal{C} \rightarrow$. It then follows that $\operatorname{Ind}(\mathcal{W})$ is a full subcategory of $\operatorname{Ind}\left(\mathcal{C}^{\rightarrow}\right)$, and thus $\operatorname{Ind}(\mathcal{W}) \simeq L w \cong(\mathcal{W})$.

We now come to our first criterion:

Proposition 4.3. Let $(\underline{\mathcal{M}}, \underline{\mathcal{W}}, \underline{\mathcal{F}}, \underline{\mathcal{C}})$ be an $\omega$-combinatorial model category. Let $\mathcal{M}$ denote the full subcategory of $\mathcal{M}$ spanned by the finitely presentable objects. Let $\mathcal{W}, \mathcal{C}$ denote the classes of weak equivalences and cofibrations between objects in $\mathcal{M}$, respectively. We denote by $L P$ the class of left proper maps in $(\mathcal{M}, \mathcal{W})$, and by $R P$ the class of right proper maps in $(\mathcal{M}, \mathcal{W})$.

We make the following further assumptions:

1. The category $\mathcal{M}$ has finite limits.

2. $\operatorname{Mor}(\mathcal{M})=\mathcal{W} \circ \mathcal{C}$.

3. $\operatorname{Mor}(\mathcal{M})=\mathcal{W} \circ L P$.

4. $\operatorname{Mor}(\mathcal{M})=R P \circ L P$. 
Then $(\mathcal{M}, \mathcal{W}, \mathcal{C})$ is an ind-admissible weak cofibration category and the induced model structure on $\operatorname{Ind}(\mathcal{M})$, given by Theorem 2.20, coincides with $(\underline{\mathcal{M}}, \underline{\mathcal{W}}, \underline{\mathcal{F}}, \underline{\mathcal{C}})$, under the natural equivalence $\mathcal{M} \simeq \operatorname{Ind}(\mathcal{M})$.

In particular, it follows from Lemma 4.2 that the full subcategory of $\mathcal{M}^{\rightarrow}$, spanned by the class of weak equivalences, is finitely accessible.

Proof. Since $\mathcal{M}$ is locally finitely presentable (being $\omega$-combinatorial), it follows that its full subcategory $\mathcal{M}$ is essentially small, and closed under finite colimits, and that we have a natural equivalence of categories $\operatorname{Ind}(\mathcal{M}) \simeq \underline{\mathcal{M}}$ given by taking colimits (see $[$ AdRo]).

It is now trivial to verify, using assumption 2 above, that $(\mathcal{M}, \mathcal{W}, \mathcal{C})$ is a weak cofibration category. Using assumptions 1, 3, and 4, we get, by Proposition 3.7, that $(\mathcal{M}, \mathcal{W}, \mathcal{C})$ is almost ind-admissible (see Definition 3.13). Thus, by Theorem 3.14, there exists an almost model category structure on $\underline{\mathcal{M}} \simeq \operatorname{Ind}(\mathcal{M})$ such that:

1. The weak equivalences are $\overline{\mathcal{W}}:=L w^{\simeq}(\mathcal{W})$.

2. The fibrations are $\overline{\mathcal{F}}:=(\mathcal{C} \cap \mathcal{W})^{\perp}$.

Furthermore, we have $\overline{\mathcal{F}} \cap \overline{\mathcal{W}}=\mathcal{C}^{\perp}$.

Since the model category $(\underline{\mathcal{M}}, \underline{\mathcal{W}}, \underline{\mathcal{F}}, \underline{\mathcal{C}})$ is $\omega$-combinatorial, we have that

$$
\begin{gathered}
\overline{\mathcal{F}} \cap \overline{\mathcal{W}}=\mathcal{C}^{\perp}=\underline{\mathcal{F}} \cap \underline{\mathcal{W}}, \\
\overline{\mathcal{F}}:=(\mathcal{C} \cap \mathcal{W})^{\perp}=\underline{\mathcal{F}} .
\end{gathered}
$$

Thus, using Lemma 3.10, we also obtain

$$
\begin{gathered}
\overline{\mathcal{C}} \cap \overline{\mathcal{W}}={ }^{\perp} \overline{\mathcal{F}}={ }^{\perp} \underline{\mathcal{F}}=\underline{\mathcal{C}} \cap \underline{\mathcal{W}}, \\
\overline{\mathcal{C}}:={ }^{\perp}(\overline{\mathcal{F}} \cap \overline{\mathcal{W}})={ }^{\perp}(\underline{\mathcal{F}} \cap \underline{\mathcal{W}})=\underline{\mathcal{C}} .
\end{gathered}
$$

It is now easy to show that $\overline{\mathcal{W}}=\underline{\mathcal{W}}$ : we will show that $\overline{\mathcal{W}} \subseteq \underline{\mathcal{W}}$, and the other direction can be shown similarly.

Let $f: X \rightarrow Y$ be an element in $\overline{\mathcal{W}}$. We decompose $f$, in the almost model category $(\underline{\mathcal{M}}, \overline{\mathcal{W}}, \overline{\mathcal{F}}, \overline{\mathcal{C}})$, into an acyclic cofibration followed by a fibration:

$$
X \stackrel{h \in \overline{\mathcal{C}} \cap \overline{\mathcal{W}}}{\longrightarrow} Z \stackrel{g \in \overline{\mathcal{F}}}{\longrightarrow} Y .
$$

Since the weak cofibration category $(\mathcal{M}, \mathcal{W}, \mathcal{C})$ is almost ind-admissible, we have that $g$ also belongs to $\overline{\mathcal{W}}$. Thus, we have

$$
\begin{aligned}
& h \in \overline{\mathcal{C}} \cap \overline{\mathcal{W}}=\underline{\mathcal{C}} \cap \underline{\mathcal{W}}, \\
& g \in \overline{\mathcal{F}} \cap \overline{\mathcal{W}}=\underline{\mathcal{F}} \cap \underline{\mathcal{W}} .
\end{aligned}
$$

It follows that $f \in \underline{\mathcal{W}}$, because $\underline{\mathcal{W}}$ is closed under composition.

We now come to our second criterion for the finite accessibility of the category of weak equivalences.

Theorem 4.4. Let $(\underline{\mathcal{M}}, \underline{\mathcal{W}}, \underline{\mathcal{F}}, \underline{\mathcal{C}})$ be an $\omega$-combinatorial left proper model category. Let $\mathcal{M}$ denote the full subcategory of $\mathcal{M}$ spanned by the finitely presentable objects. Let $\mathcal{W}, \mathcal{C}$ denote the classes of weak equivalences and cofibrations between objects in $\mathcal{M}$, respectively. 
Suppose we are given a cylinder object in $\mathcal{M}$; that is, for every object $B$ of $\mathcal{M}$ we are given a factorization in $\mathcal{M}$ of the fold map $B \sqcup B \rightarrow B$ into a cofibration followed by a weak equivalence:

$$
B \sqcup B \stackrel{\left(i_{0}, i_{1}\right)}{\longrightarrow} I \otimes B \stackrel{p}{\rightarrow} B .
$$

(Note that we are not assuming any simplicial structure; $I \otimes B$ is just a suggestive notation.)

We make the following further assumptions:

1. The category $\mathcal{M}$ has finite limits.

2. Every object in $\mathcal{M}$ is cofibrant.

3. For every morphism $f: A \rightarrow B$ in $\mathcal{M}$, the map $B \coprod_{A}(I \otimes A) \rightarrow B$, induced by the commutative square

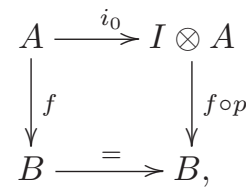

is a right proper map in $(\mathcal{M}, \mathcal{W})$.

Then $(\mathcal{M}, \mathcal{W}, \mathcal{C})$ is an ind-admissible weak cofibration category and the induced model structure on $\operatorname{Ind}(\mathcal{M})$, given by Theorem 2.20, coincides with $(\underline{\mathcal{M}}, \underline{\mathcal{W}}, \underline{\mathcal{F}}, \underline{\mathcal{C}})$, under the natural equivalence $\underline{\mathcal{M}} \simeq \operatorname{Ind}(\mathcal{M})$.

In particular, it follows from Lemma 4.2 that the full subcategory of $\underline{\mathcal{M}^{\rightarrow}}$, spanned by the class of weak equivalences, is finitely accessible.

Proof. We will verify that all the conditions of Proposition 4.3 are satisfied. We only need to check the existence of factorizations of the form

1. $\operatorname{Mor}(\mathcal{M})=\mathcal{W} \circ \mathcal{C}$,

2. $\operatorname{Mor}(\mathcal{M})=\mathcal{W} \circ L P$,

3. $\operatorname{Mor}(\mathcal{M})=R P \circ L P$.

All the factorizations above will be given by the same factorization, which we now describe. This is just the mapping cylinder factorization relative to our given cylinder object for $\mathcal{M}$.

It is not hard to show that for any $B \in \mathcal{M}$ the maps $i_{0}, i_{1}: B \rightarrow I \otimes B$ are acyclic cofibrations.

Let $f: A \rightarrow B$ be a morphism in $\mathcal{M}$. We define the mapping cylinder of $f$ to be the push-out

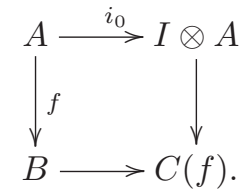

We define a morphism $q: C(f)=B \coprod_{A}(I \otimes A) \rightarrow B$ to be the one induced by the 
commutative square

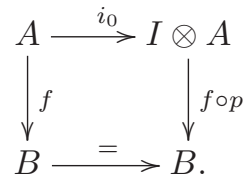

We define a morphism $i: A \rightarrow C(f)=B \coprod_{A}(I \otimes A)$ to be the composition

$$
A \stackrel{i_{1}}{\rightarrow} I \otimes A \rightarrow C(f) .
$$

Clearly $f=q i$, and we call this the mapping cylinder factorization.

The map $q$ is a left inverse to $j$, defined by the mapping cylinder push-out square

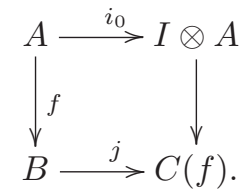

Since $i_{0}$ is an acyclic cofibration, we get that $j$ is also an acyclic cofibration and, in particular, $q$ is a weak equivalence.

The map $i$ is a cofibration, being a composite of two cofibrations

$$
A \longrightarrow B \coprod A \stackrel{(j, i)}{\longrightarrow} B \coprod_{A}(I \otimes A)
$$

These maps are cofibrations because of the following push-out squares:
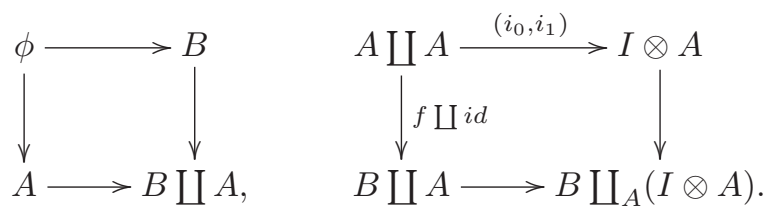

Since the map $i$ is a cofibration and $\underline{\mathcal{M}}$ is left proper, we get that the map $i$ is also left proper. By assumption 3, $q$ is right proper.

We now come to our third and last criterion.

Theorem 4.5. Let $(\underline{\mathcal{M}}, \underline{\mathcal{W}}, \underline{\mathcal{F}}, \underline{\mathcal{C}})$ be an $\omega$-combinatorial left proper model category. Let $\mathcal{M}$ denote the full subcategory of $\mathcal{M}$ spanned by the finitely presentable objects. Assume that the category $\mathcal{M}$ has finite limits, and let $*$ denote the terminal object in $\mathcal{M}$. Let $\mathcal{W}, \mathcal{C}$ denote the classes of weak equivalences and cofibrations between objects in $\mathcal{M}$, respectively.

Suppose we are given a factorization in $\mathcal{M}$ of the fold map $* \sqcup * \rightarrow *$ into a cofibration followed by a weak equivalence:

$$
* \sqcup * \rightarrow I \rightarrow *
$$

We make the following further assumptions: 
1. For every morphism $Y \rightarrow B$ in $\mathcal{M}$, the functor

$$
Y \times_{B}(-): \mathcal{M}_{/ B} \rightarrow \mathcal{M}
$$

commutes with finite colimits.

2. Every object in $\mathcal{M}$ is cofibrant.

3. For every object $B$ in $\mathcal{M}$, the functor

$$
B \times(-): \mathcal{M} \rightarrow \mathcal{M}
$$

preserves cofibrations and weak equivalences.

Then $(\mathcal{M}, \mathcal{W}, \mathcal{C})$ is an ind-admissible weak cofibration category and the induced model structure on $\operatorname{Ind}(\mathcal{M})$, given by Theorem 2.20, coincides with $(\underline{\mathcal{M}}, \underline{\mathcal{W}}, \underline{\mathcal{F}}, \underline{\mathcal{C}})$, under the natural equivalence $\underline{\mathcal{M}} \simeq \operatorname{Ind}(\mathcal{M})$.

In particular, it follows from Lemma 4.2 that the full subcategory of $\underline{\mathcal{M}}^{\rightarrow}$, spanned by the class of weak equivalences, is finitely accessible.

Proof. We will verify that all the conditions of Theorem 4.4 are satisfied. For every object $B$ of $\mathcal{M}$, we have that the induced diagram

$$
B \sqcup B \cong(* \times B) \sqcup(* \times B) \cong(* \sqcup *) \times B \rightarrow I \times B \rightarrow * \times B \cong B
$$

is a factorization in $\mathcal{M}$ of the fold map $B \sqcup B \rightarrow B$ into a cofibration followed by a weak equivalence. (Note that here $\times$ denotes the actual categorical product and is not just a suggestive notation.)

Thus, we only need to check that for every morphism $f: A \rightarrow B$ in $\mathcal{M}$ the map $q: B \coprod_{A}(I \times A) \rightarrow B$, induced by the commutative square

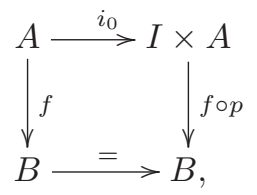

is a right proper map in $(\mathcal{M}, \mathcal{W})$.

We will use the same notation as in the proof of Theorem 4.4, regarding the mapping cylinder factorization.

Let

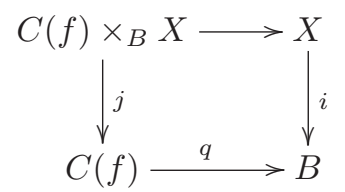

be a pull-back square in $\mathcal{M}$ such that $i$ is a weak equivalence. We need to show that $j$ is a weak equivalence. Using condition 1 , we get natural isomorphisms

$$
\begin{gathered}
C(f) \times_{B} X=\left(B \coprod_{A}(I \times A)\right) \times_{B} X \cong\left(B \times_{B} X\right) \coprod_{A \times_{B} X}\left((I \times A) \times_{B} X\right) \cong \\
\cong\left(X \coprod_{A \times_{B} X}\left(I \times\left(A \times_{B} X\right)\right)=C(k),\right.
\end{gathered}
$$


where $k: A \times_{B} X \rightarrow X$ is the natural map. By condition 3 and the proof of Theorem 4.4, we get that the natural map $C(k) \cong C(f) \times_{B} X \rightarrow X$ is a weak equivalence. By the two-out-of-three property, we get that $j$ is also a weak equivalence.

We now turn to our main example:

Theorem 4.6. Let $\mathcal{S}$ denote the category of simplicial sets with its standard model structure. Let $\mathcal{S}_{f}$ denote the full subcategory of $\mathcal{S}$ spanned by the finitely presentable objects. Let $\mathcal{W}, \mathcal{C}$ denote the classes of weak equivalences and cofibrations between objects in $\mathcal{S}_{f}$, respectively.

Then $\left(\mathcal{S}_{f}, \mathcal{W}, \mathcal{C}\right)$ is an ind-admissible weak cofibration category and the induced model structure on $\operatorname{Ind}\left(\mathcal{S}_{f}\right)$, given by Theorem 2.20, coincides with the standard model structure on $\mathcal{S}$, under the natural equivalence $\mathcal{S} \simeq \operatorname{Ind}\left(\mathcal{S}_{f}\right)$.

In particular, it follows from Lemma 4.2 that the full subcategory of $\mathcal{S} \rightarrow$, spanned by the class of weak equivalences, is finitely accessible.

Proof. We will verify that all the conditions of Theorem 4.5 are satisfied. The model category $\mathcal{S}$ is $\omega$-combinatorial and left proper.

We first sketch a proof showing that the subcategory $\mathcal{S}_{f}$ of $\mathcal{S}$ is closed under finite limits.

Let $X$ be a finite simplicial set. It is not hard to verify that there exists a finite diagram $F: D \rightarrow\left\{\Delta^{0}, \Delta^{1}, \Delta^{2}, \ldots\right\}$ such that

$$
X \cong \operatorname{colim}_{D} F .
$$

We now note the following facts:

1. In the category $\mathcal{S}$, pull-backs commute with colimits.

2. For all $n, m \geqslant 0, \Delta^{n} \times \Delta^{m}$ belongs to $\mathcal{S}_{f}$ (by direct computation).

3. A sub-simplicial set of a finite simplicial set is also finite.

4. The colimit in $\mathcal{S}$, of a finite diagram in $\mathcal{S}_{f}$, belongs to $\mathcal{S}_{f}$.

Using these facts, it is not hard to check that the pull-back (in $\mathcal{S}$ ) of objects in $\mathcal{S}_{f}$ belongs to $\mathcal{S}_{f}$. Since the terminal object in $\mathcal{S}$ also belongs to $\mathcal{S}_{f}$, it follows that the subcategory $\mathcal{S}_{f}$ of $\mathcal{S}$ is closed under finite limits.

In particular, this shows that $\mathcal{S}_{f}$ admits finite limits and that they can be calculated in $\mathcal{S}$. This also gives condition 1 of Theorem 4.5 (as this condition is known to hold in $\mathcal{S})$.

Clearly every object in $\mathcal{S}_{f}$ is cofibrant, so condition 2 is satisfied.

Let $B$ be an object in $\mathcal{S}_{f}$. Since $B$ is cofibrant and $\mathcal{S}$ is a simplicial model category, we get that the functor

$$
B \times(-): \mathcal{S} \rightarrow \mathcal{S}
$$

is a left Quillen functor and thus preserves cofibrations and weak equivalences between cofibrant objects. Since every object in $\mathcal{S}_{f}$ is cofibrant, we get that

$$
B \times(-): \mathcal{S}_{f} \rightarrow \mathcal{S}_{f}
$$

preserves cofibrations and weak equivalences. This gives condition 3 . 
Finally, we may take the factorization of the fold map,

$$
* \sqcup * \rightarrow I \rightarrow *,
$$

to be $\Delta^{\{0\}} \sqcup \Delta^{\{1\}} \rightarrow \Delta^{1} \rightarrow \Delta^{0}$.

Remark 4.7. Let $f: X \rightarrow Y$ be a morphism in $\mathcal{S}_{f}$. In the proof of Theorem 4.4, we considered the mapping cylinder factorization of $f: X \stackrel{h}{\rightarrow} C(f) \stackrel{g}{\rightarrow} Y$. We showed that $g$ is right proper. Note that $g$ is not, in general, a fibration in $\mathcal{S}$. Consider the map $f: \Delta^{n} \rightarrow \Delta^{0}(n \geqslant 0)$. Then the mapping cylinder factorization of $f$ is just $\Delta^{\{1, \ldots, n+1\}} \rightarrow \Delta^{n+1} \rightarrow \Delta^{0}$. But $\Delta^{n+1} \rightarrow \Delta^{0}$ is not a Kan fibration, since $\Delta^{n+1}$ is not a Kan complex. Thus, we see that we are using the extra generalization provided by Proposition 3.7 over Isaksen's results [Isa1, Lemmas 3.5 and 3.6].

\section{Appendix A. Relation to the work of Raptis and Rosický}

In this paper, we proved theorems giving sufficient conditions for the finite accessibility of the category of weak equivalences in combinatorial model categories. Our main application was to the standard model structure on the category of simplicial sets, deducing the finite accessibility of its class of weak equivalences. As mentioned in the introduction, the same result on simplicial sets was also proved in [RaRo], using different methods. In this appendix, we explain a possible connection between the two approaches.

An important ingredient in the proof of [RaRo] is a generalization of Quillen's small-object argument (called the fat small-object argument). Our proof is based mainly on Theorem 2.20, describing a construction of a model structure on the indcategory of an essentially small weak cofibration category. Theorem 2.20 was not proved directly, but was deduced, by duality, from Theorem 2.18. The main technical tool in the proof of Theorem 2.18 is a certain factorization proposition, namely, [BaSc2, Proposition 3.18]. The main purpose of this appendix is to prove Proposition A.11, which connects the notion of a relative cell complex, appearing in Quillen's small-object argument, and the notion of an essentially cospecial map, appearing in Proposition A.6 (which is the dual version of [BaSc2, Proposition 3.18], and which is used in proving Theorem 2.20). This will hopefully shed some light as to possible connections between the approach taken in this paper and that of Raptis and Rosický.

As we explain below, Proposition A.11 solves a conjecture of Isaksen. We end the appendix with an application of Proposition A.11 to finite simplicial sets.

Definition A.1. Let $T$ be a poset. Then we view $T$ as a category that has a single morphism $u \rightarrow v$ iff $u \leqslant v$. Note that this convention is the opposite of that used in $[\mathrm{BaSc2}]$.

Thus, a poset $T$ is filtered (see Definition 2.8) iff $T$ is non-empty and for every $a, b$ in $T$ there exists an element $c$ in $T$ such that $c \geqslant a, b$. A filtered poeset will also be called directed.

Definition A.2. A cofinite poset is a poset $T$ such that for every element $x$ in $T$ the set $T_{x}:=\{z \in T \mid z \leqslant x\}$ is finite. 
Definition A.3. Let $\mathcal{C}$ be a category with finite colimits, $N$ a class of morphisms in $\mathcal{C}, I$ a cofinite poset (see Definition A.2), and $F: X \rightarrow Y$ a morphism in $\mathcal{C}^{I}$. Then the map $F$ will be called a cospecial $N$-map if the natural map

$$
X_{t} \coprod_{\operatorname{colim}_{s<t} X_{s}} \operatorname{colim}_{s<t} Y_{s} \rightarrow Y_{t}
$$

is in $N$, for every $t$ in $I$. We will denote this by $F \in \operatorname{coSp}(N)$.

Definition A.4. Let $\mathcal{C}$ be a category, and let $N$ be a class of morphisms in $\mathcal{C}$.

1. We denote by $R(N)$ the class of morphisms in $\mathcal{C}$ that are retracts of morphisms in $N$. Note that $R(R(N))=R(N)$.

2. If $\mathcal{C}$ has finite colimits, we denote by $\operatorname{coSp} \cong(N)$ the class of morphisms in $\operatorname{Ind}(\mathcal{C})$ that are isomorphic to a morphism that comes from a natural transformation which is a cospecial $N$-map (see Definition A.3). Maps in $\operatorname{coSp} \cong(N)$ are called essentially cospecial $N$-maps.

In the following, we bring a few results from several papers. These results were originally stated in the language of pro-categories. For the convenience of the reader, we bring them in their dual formulation, which we need here.

Proposition A.5 ([BaSc2, Proposition 2.19]). Let $\mathcal{C}$ be a category with finite colimits, and let $\mathcal{N} \subseteq \mathcal{C}$ be a subcategory that is closed under cobase change and contains all the isomorphisms. Let $F: X \rightarrow Y$ be a natural transformation between diagrams in $\mathcal{C}$, which is a cospecial $\mathcal{N}$-map. Then $F$ is a levelwise $\mathcal{N}$-map.

We now state our factorization proposition, which is the main technical tool in the proof of Theorem 2.20 .

Proposition A.6 ([BaSc2, Proposition 3.18]). Let $\mathcal{C}$ be an essentially small category that has finite colimits, $\mathcal{N} \subseteq \mathcal{C}$ a subcategory that is closed under cobase change, and $M \subseteq \operatorname{Mor}(\mathcal{C})$ an arbitrary class of morphisms such that $M \circ \mathcal{N}=\operatorname{Mor}(\mathcal{C})$.

Then every morphism $f: X \rightarrow Y$ in $\operatorname{Ind}(\mathcal{C})$ can be functorially factored as $X \stackrel{g}{\rightarrow}$ $H_{f} \stackrel{h}{\rightarrow} Y$, where $g$ is in $\operatorname{coSp} \cong(\mathcal{N})$ and $h$ is in $L w \cong(M) \cap \mathcal{N}^{\perp}$.

We can now also state the more elaborate version of Theorem 2.20, and the proof is exactly the same.

Theorem A.7. Let $(\mathcal{C}, \mathcal{W}, \mathcal{C}$ of $)$ be an essentially small ind-admissible weak cofibration category. Then there exists a model category structure on $\operatorname{Ind}(\mathcal{C})$ such that:

1. The weak equivalences are $\mathbf{W}:=L w^{\cong}(\mathcal{W})$.

2. The cofibrations are $\mathbf{C}:=R(\operatorname{coSp} \cong(\mathcal{C} \circ f))$.

3. The fibrations are $\mathbf{F}:=(\operatorname{Cof} \cap \mathcal{W})^{\perp}$.

Moreover, this model category is $\omega$-combinatorial, with set of generating cofibrations $\mathcal{C}$ of and set of generating acyclic cofibrations $\operatorname{Cof} \cap \mathcal{W}$. Furtheremore, the acyclic cofibrations in this model structure are given by

$$
\mathbf{C} \cap \mathbf{W}=R(\operatorname{coSp} \cong(\mathcal{C} \circ \cap \mathcal{W})) .
$$

The following two definitions are based on [Hov, Section 2.1]. 
Definition A.8. Let $\mathcal{D}$ be a category with all small colimits, $N \subseteq \operatorname{Mor}(\mathcal{D})$ a class of morphisms in $\mathcal{D}$, and $\lambda$ an ordinal. A $\lambda$-sequence in $\mathcal{D}$, relative to $N$, is a diagram $X: \lambda \rightarrow \mathcal{D}$, such that for all limit ordinals $t<\lambda$ the natural map $\operatorname{colim}_{s<t} X_{s} \rightarrow X_{t}$ is an isomorphism, and for all non-limit ordinals $t<\lambda$, the map $X_{t-1} \rightarrow X_{t}$ is in $N$. The (transfinite) composition of the $\lambda$-sequence $X$ is defined to be the natural map $X(0) \rightarrow \operatorname{colim}_{\lambda} X$.

Definition A.9. Let $\mathcal{D}$ be a category with all small colimits, and let $N \subseteq \operatorname{Mor}(\mathcal{D})$ be a class of morphisms in $\mathcal{D}$. A relative $N$-cell complex, is a transfinite composition of push-outs of elements of $N$. That is, $f: A \rightarrow B$ is a relative $N$-cell complex if there exists an ordinal $\lambda$, and a $\lambda$-sequence in $\mathcal{D}$, relative to push-outs of maps in $N$, such that $f$ is isomorphic to the composition of $X$. We denote the collection of all relative $N$-cell complexes by $\operatorname{cell}(N)$.

From now until the end of this section, we let $\mathcal{C}$ be an essentially small category with finite colimits. By the results of $[\mathbf{A d R o}]$, the category $\operatorname{Ind}(\mathcal{C})$ is locally presentable and every object of $\mathcal{C}$ is $\omega$-presentable in $\operatorname{Ind}(\mathcal{C}) . \operatorname{In}$ particular, the category $\operatorname{Ind}(\mathcal{C})$ has all small colimits.

Proposition A.10 ([Isa1, Proposition 5.2]). For any class of morphisms $N \subseteq \operatorname{Mor}(\mathcal{C})$, we have $\operatorname{coSp} \cong(N) \subseteq \operatorname{cell}(N)$, in $\operatorname{Ind}(\mathcal{C})$.

In [Isa1], Isaksen conjectures a partial converse to Proposition A.10. Namely, that for any class of morphisms $N \subseteq \operatorname{Mor}(\mathcal{C})$, we have $R(\operatorname{cell}(N)) \subseteq R\left(\operatorname{coS} S p^{\simeq}(N)\right)$, in $\operatorname{Ind}(\mathcal{C})$. This conjecture fails as stated, as the following counterexample demonstrates. Take $\mathcal{C}$ to be the category

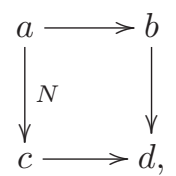

where the square is commutative, and take $N$ to consist only of the unique map $a \rightarrow c$. It is easy to verify that there is a natural equivalence of categories $\operatorname{Ind}(\mathcal{C}) \simeq \mathcal{C}$, and that under this equivalence $R(\operatorname{coSp} \cong(N))$ is just $N$. Thus, the unique map $b \rightarrow d$ belongs to $R(\operatorname{cell}(N))$ but not to $R(\operatorname{coSp} \cong(N))$.

However, using Theorem 2.20, we can prove Isaksen's conjecture in the case where $N$ is a subcategory that is closed under cobase change.

Proposition A.11. Let $\mathcal{N} \subseteq \mathcal{C}$ be a subcategory that is closed under cobase change and contains all the isomorphisms. Then $R(\operatorname{cell}(\mathcal{N}))=R(\operatorname{coSp} \cong(\mathcal{N}))$.

Proof. By Proposition A.10, we know that $R(\operatorname{coSp} \cong(\mathcal{N})) \subseteq R(\operatorname{cell}(\mathcal{N}))$. It thus remains to show that $R(\operatorname{cell}(\mathcal{N})) \subseteq R(\operatorname{coSp} \cong(\mathcal{N}))$.

Since $\mathcal{N} \subseteq R(\operatorname{coSp} \cong(\mathcal{N}))$, it is enough to show that the class $R(\operatorname{coSp} \cong(\mathcal{N})) \subseteq$ $\operatorname{Mor}(\operatorname{Ind}(\mathcal{C}))$ is closed under cobase change and transfinite compositions.

It is easy to see that $(\mathcal{C}, \mathcal{C}, \mathcal{N})$ is an essentially small weak cofibration category. Moreover, $L w^{\simeq}(\mathcal{C})=\operatorname{Mor}(\operatorname{Ind}(\mathcal{C}))$ by (the dual version of) Lemma 2.5 , so $(\mathcal{C}, \mathcal{C}, \mathcal{N})$ is clearly ind-admissible. Thus, it follows from Theorem A.7 that there exists a model category structure on $\operatorname{Ind}(\mathcal{C})$ such that: 
1. The weak equivalences are $\mathbf{W}:=L w \cong(\mathcal{C})=\operatorname{Mor}(\operatorname{Ind}(\mathcal{C}))$.

2. The cofibrations are $\mathbf{C}:=R\left(\operatorname{coS} p^{\cong}(\mathcal{N})\right)$.

3. The fibrations are $\mathbf{F}:=\mathcal{N}^{\perp}$.

In particular, it follows that

$$
R(\operatorname{coSp} \cong(\mathcal{N}))={ }^{\perp}(\mathbf{F} \cap \mathbf{W})={ }^{\perp}\left(\mathcal{N}^{\perp}\right),
$$

and thus $R(\operatorname{coSp} \cong(\mathcal{N}))$ is closed under cobase change and transfinite compositions by well-known arguments (see, for example, [Lur, Section A.1.1]).

Proposition A.11 can be used to compare Quillen's small-object argument with our factorization proposition (Proposition A.6). As an example, we show how a special case of the small-object argument follows easily from our factorization proposition.

Corollary A.12. Let $N \subseteq \operatorname{Mor}(\mathcal{C})$ be any class of morphisms. Then every map $f$ : $X \rightarrow Y$ in $\operatorname{Ind}(\mathcal{C})$ can be functorially factored as $X \stackrel{h}{\rightarrow} H \stackrel{g}{\rightarrow} Y$, where $g$ is in cell $(N)$ and $h$ is in $N^{\perp}$.

Proof. Let $\mathcal{N}$ denote the smallest subcategory of $\mathcal{C}$ that is closed under cobase change, contains all the isomorphisms, and also contains $N$. Since the classes $\operatorname{cell}(N)$ and ${ }^{\perp}\left(N^{\perp}\right)$ are closed under cobase change and transfinite composition, we have:

1. $\operatorname{cell}(N)=\operatorname{cell}(\mathcal{N})$.

2. $N^{\perp}=\left({ }^{\perp}\left(N^{\perp}\right)\right)^{\perp}=\mathcal{N}^{\perp}$.

Thus, the corollary follows by combining Propositions A.6 and A.11.

We now present a nice application of Proposition A.11. Let $\mathcal{S}_{f}$ denote the category of simplicial sets with finitely many non-degenerate simplices. Let $\mathcal{A}$ denote the smallest subcategory of $\mathcal{S}_{f}$ that contains all the isomorphisms, is closed under pushouts, and also contains all the horn inclusions $\Lambda_{i}^{n} \rightarrow \Delta^{n}$. In other words, if $H$ denotes the set of horn inclusions, then maps in $\mathcal{A}$ are just finite relative $H$-cell complexes in $\mathcal{S}_{f}$. That is, maps that can be obtained as a finite composition of push outs of horn inclusions, starting from an arbitrary object in $\mathcal{S}_{f}$. Clearly, every map in $\mathcal{A}$ is a trivial cofibration in $\mathcal{S}_{f}$.

Proposition A.13. Every trivial cofibration in $\mathcal{S}_{f}$ is a retract of a map in $\mathcal{A}$.

Proof. Let $f: A \rightarrow B$ be a trivial cofibration in $\mathcal{S}_{f}$. By the results of [Hov, Section 2.1], $f$ belongs to $R(\operatorname{cell}(H))=R(\operatorname{cell}(\mathcal{A}))$ as a map in $\operatorname{Ind}\left(\mathcal{S}_{f}\right) \simeq \mathcal{S}$. By Proposition A.11, $f$ also belongs to $R\left(\operatorname{coS} p^{\cong}(\mathcal{A})\right)$. Thus, there exists $h \in \operatorname{coS} p^{\cong}(\mathcal{A})$ such that $f$ is a retract of $h$. Without loss of generality, we may assume that $h:\left\{X_{t}\right\}_{t \in T} \rightarrow$ $\left\{Y_{t}\right\}_{t \in T}$ is a natural transformation, which is a cospecial $\mathcal{A}$-map. We have the following retract diagram:

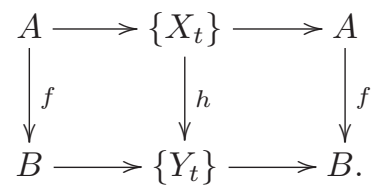

It follows from the definition of morphisms in $\operatorname{Ind}\left(\mathcal{S}_{f}\right)$ that there exists $t_{0} \in T$ such 
that the above diagram can be factored as

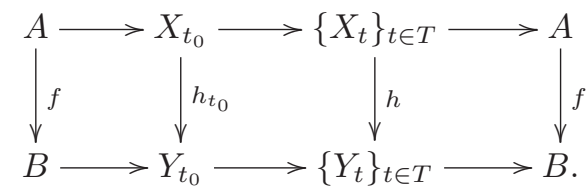

It follows that $f$ is a retract of $h_{t_{0}}$, in $\mathcal{S}_{f}$. But by Proposition A.5, $h$ is a levelwise $\mathcal{A}$-map. In particular, $h_{t_{0}}$ belongs to $\mathcal{A}$, and we get the desired result.

\section{References}

[ArMa] M. Artin and B. Mazur, Étale Homotopy, Lecture Notes in Mathematics, Vol. 100, Springer-Verlag, Berlin, 1969.

[AdRo] J. Adamek and J. Rosický, Locally Presentable and Accessible Categories, Cambridge University Press, Cambridge, 1994.

[BaKa] C. Barwick and D.M. Kan, Relative categories: Another model for the homotopy theory of homotopy theories, preprint, available at http:// arxiv.org/abs/1011.1691.

[BaSc1] I. Barnea and T.M. Schlank, A new model for pro-categories, J. Pure Appl. Algebra 219, no. 4 (2015), 1175-1210.

[BaSc2] I. Barnea and T.M. Schlank, A projective model structure on pro simplicial sheaves, and the relative étale homotopy type, To appear in Adv. Math., 2015. Preprint available at http://arxiv.org/abs/1109.5477.

[Rad] A. Radulescu-Banu, Cofibrations in homotopy theory, preprint, available at http://arxiv.org/abs/math/0610009.

[Bau] H.J. Baues, Algebraic Homotopy, Cambridge University Press, Cambridge, 1988.

[Bro] K.S. Brown, Abstract homotopy theory and generalized sheaf cohomology, Trans. Amer. Math. Soc. 186 (1973), 419-458, available at http:/ /arxiv.org/abs/math/0610439.

[Hov] M. Hovey Model Categories, Mathematical Surveys and Monographs Vol. 63, AMS, Providence, RI, 1998.

[Isa1] D.C. Isaksen, Strict model structures for pro-categories, Categorical Factorization Techniques in Algebraic Topology (Isle of Skye, 2001), 179-198, Progr. Math. 215, Birkhäuser, Basel, 2004.

[Isa2] D.C. Isaksen, Calculating limits and colimits in pro-categories, Fund. Math. 175, no. 2 (2002), 175-194.

[Lur] J. Lurie, Higher Topos Theory, Annals of Mathematics Studies, Vol. 170, Princeton University Press, Princeton, NJ, 2009.

[Qui] D.G. Quillen, Homotopical Algebra, Lecture Notes in Mathematics, Vol. 43, Springer-Verlag, Berlin, 1967.

[RaRo] G. Raptis and J. Rosický, The accessibility rank of weak equivalences, preprint, available at http://arxiv.org/abs/1403.3042. 
[Rez] C. Rezk, Fibrations and homotopy colimits of simplicial sheaves, preprint, available at http://www.math.uiuc.edu/ rezk/rezk-sharp-maps.pdf.

Ilan Barnea ilanbarnea770@gmail.com

Department of Mathematics, University of Münster, Einsteinstrasse 62, Münster, Nordrhein-Westfalen, 48149, Germany

Tomer M. Schlank schlank@math.mit.edu

Department of Mathematics, Massachusetts Institute of Technology, 50 Ames Street, Cambridge, MA 02142, USA 